\title{
Biofunctionalization of cellulosic fibres with L-cysteine: Assessment of antibacterial properties and mechanism of action against Staphylococcus aureus and Klebsiella pneumoniae
}

\author{
Estela Caldeira ${ }^{\mathrm{a}}$, Erhan Piskin ${ }^{\mathrm{b}}$, Luiza Granadeiro ${ }^{\mathrm{c}}$, Filomena Silva ${ }^{\mathrm{c}}$, Isabel C. Gouveia ${ }^{\mathrm{a}, *}$ \\ ${ }^{a} R E D D$ Unit of Textile and Paper Materials, Faculty of Engineering, University of Beira Interior, 6201-001 Covilhã, Portugal \\ ${ }^{\mathrm{b}}$ Center for Bioengineering and Biyomedtek, Hacettepe University, Ankara, Turkey

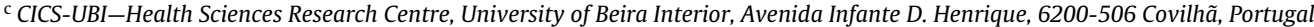

\section{A R T I C L E I N F O}

\section{Article history:}

Received 21 April 2013

Received in revised form 6 October 2013

Accepted 14 October 2013

Available online 25 October 2013

\section{Keywords:}

Antimicrobial-textiles

Cellulose

Antimicrobial agents

Staphylococcus aureus

Klebsiella pneumoniae

Flow cytometry

\begin{abstract}
A B S T R A C T
The main purpose of this work is to obtain a cotton-based textile material functionalized with L-cysteine (L-cys) to achieve an antimicrobial effect with potential application in biomedical, geriatric or pediatric textiles. The binding capacity of L-cys to cotton fibres was assessed through different functionalization strategies-surface activation and exhaustion processes. A subsequent analysis of the possible antibacterial action against Staphylococcus aureus and Klebsiella pneumoniae was performed according with the Japanese International standard (JISL, 2008). To determine the mechanism of action of L-cys on the selected strains, flow cytometry was used.

The results revealed that the exhaustion process was performed with success to confer bioactivity to the treated fabric, as assessed by an effective antibacterial effect against both Gram-positive and Gram-negative bacteria, and successfully linkage of L-cys was observed via FTIR with a durable effect demonstrated after the washing tests (fastness to washing). It was also observed that L-cys exerts a bacteriostatic effect against both bacterial strains, since there were alterations in the metabolic activity of the microorganisms after the application of the bioactive textile which was shown by the CTC (cyanoditolyl tetrazolium chloride) staining used in flow cytometry.

This study shows a new and successful biotechnological process to develop antibacterial textiles through the functionalization of cotton fibres with L-cys which presents a broad range of applications in healthcare, since L-cys is a natural antibacterial compound, non-toxic and affects pathogenic bacteria related to hospital infections.
\end{abstract}

(C) 2013 Elsevier B.V. All rights reserved.

\section{Introduction}

Cotton is one of the most important natural fibres used in textile industry (Son et al., 2006). Cellulose fibres have a wide range of applications as medical textiles due to cellulose unique characteristics such as high moisture and liquids absorbency, a low content of impurities, antistatic properties, biocompatibility, biodegradability and good mechanical properties. However, cotton, as well as other natural fibres, is an excellent surface for the growth and development of microorganisms (Ristić et al., 2011).

Due to their molecular structure and a wide active surface, cellulose fibres are also a good matrix to develop bioactive and biocompatible smart materials, being an attractive material for

\footnotetext{
* Corresponding author. Tel.: +351275242053/+351275319825/+351914893625; fax: +351275349725 .

E-mail address: igouveia@ubi.pt (I.C. Gouveia).
}

antimicrobial functionalization. Surface modification of cellulose fibres is currently considered the best way to provide functionality on textiles for the use in medical applications (Ristic et al., 2011). Additionally, the textile industry constantly looks for ecofriendly processes that can substitute currently in use toxic textile chemicals (Lim and Hudson, 2003).

Many of the most important classes of antimicrobial agents are used in the textile industry. Generally, these agents per se are not new and have been applied in various industries. However, their adhesion to the textile surface or incorporation into the fibres reduces their activity and limits their availability (Gouveia, 2010). In addition, their properties may be lost gradually during the use and washing cycles (Gao and Craston, 2008). For these reasons, it is necessary to apply large quantities of those products in textiles in order to control bacterial growth and maintain antimicrobial properties during use or after-laundry (Gao and Craston, 2008; Gouveia, 2010). Some of the agents commonly used in this type of textile materials are chemical 
compounds that present some risk to the environment and to the human body. The main compounds used as antimicrobial agents in textile materials are metals (like silver) and metallic salts, quaternary ammonium compounds, polyhexamethylen biguanide (PHMB), triclosan, $\mathrm{N}$-halamine compounds, peroxides and certain synthetic dyes (Gao and Craston, 2008; Gouveia, 2010; Ristić et al., 2011). In addition, the use of synthetic chemicals can raise several undesirable consequences, in particular, the potential contamination of the effluents. Thus, to overcome these limitations, the development of new techniques and processes that use antimicrobial agents of natural origin has increased not only due to their high biodegradability but also due to the possible reduction in the adverse reactions caused to the users, the environment and the materials (Schindler and Hauser, 2005; Borkow and Gabbay, 2008; Ristić et al., 2011). Some of these compounds have other beneficial properties such as antioxidant activity, which in association with antimicrobial properties can be considered a benefit in the development of medical textiles (anti-inflammatory and anti-cancer) and in the control of free radical levels, preventing adverse reactions. The most important antimicrobial natural compounds employed are chitosan, natural dyes, aloe vera, essential oils and some plant extracts, honey, and amino acids (Ramachandran et al., 2004; Gao and Craston, 2008; Joshi et al., 2009; Gouveia, 2010). However, further investigation is required to obtain effective and durable processes.

Cysteine (cys) is an uncharged polar amino acid with the polarity of its R groups being due to their sulfhydryl groups. The sulfhydryl groups play an essential role in the structure of several proteins by the formation of covalent bonds between parts of a polypeptide molecule or between two different polypeptide chains. The presence of sulfhydryl group where hydrogen can be easily replaced by radicals makes it possible to form a covalent bond with other molecules. Disulfide bridge formation between two cysteines is an example of such activity that can occur between cys residues of the peptides and proteins (Nelson and Cox, 2008). This amino acid is involved in many metabolic processes either directly or by its derivatives (Nelson and Cox, 2008). As cys is a highly reactive compound, it is not surprising that it affects several enzymatic reactions in vitro and a large number of microorganisms in various metabolic functions. Apparently, the above-described effects are responsible for cys action against microorganisms, as a potential antimicrobial agent but the phenomenon is still unclear (Kari et al., 1971; Ankri and Mirelman, 1999). Previous studies on the use of L-cys as a potential antimicrobial activity for wool has been assessed within our research group (Gouveia et al., 2011). In this study, an exhaustion method was applied to bind L-cys onto wool fibres through electrostatic interactions and antimicrobial activity was achieved for a high concentration of L-cys. However, investigation over other fibres wider applied in hospital and medical textiles is required.

Therefore, in order to develop a new cotton-based textile with antimicrobial properties being effective and non-toxic as well as using an eco-friendly process, in comparison with previous investigation in the area, this study focused on the assessment of a new biofunctionalization method of cotton fibres using natural biocide agents, in particular L-cys, to obtain an effective and non-toxic antimicrobial cotton-material with a broad application in health-care. The antimicrobial activity against Gram negative and Gram positive bacteria of L-cys alone and the resulting L-cys functionalized textile was determinated using the appropriated guidelines. Moreover, to elucidate the antibacterial action of L-cys and functionalized textiles, a flow cytometric study using fluorescent labels for identifying cellular functions was performed in order to establish the mechanism of action of L-cys on microorganisms.

\section{Materials and methods}

\subsection{Determination of L-cys minimal inhibitory concentration}

The minimal inhibitory concentration (MIC) against Staphylococcus aureus (ATCC 6538) and Klebsiella pneumoniae (ATCC11296) was determined using the broth macrodilution method, as described by CLSI M7-A6 standard method (International standard CLSI M7-A6, 2003)). According to the guidelines, the minimal inhibitory concentration was determined by serial dilution (1:2) in Mueller-Hinton Broth (MHB) (Sigma-Aldrich) with concentrations of L-cys (SAFC) ranging from $9.7 \times 10^{-2} \mathrm{~g} / \mathrm{mL}$ to $2.3 \times 10^{-5} \mathrm{~g} / \mathrm{mL}$

The inoculums were prepared from fresh overnight liquid cultures and the turbidity was adjusted to $0.5 \mathrm{McFarland}$ (approximately $1 \times 10^{8} \mathrm{CFU} / \mathrm{mL}$ ) with $0.85 \%(\mathrm{w} / \mathrm{v}) \mathrm{NaCl}$ (Sigma Aldrich), and then diluted to give a final concentration of $1 \times 10^{5} \mathrm{CFU} / \mathrm{mL}$.

A volume of $1 \mathrm{~mL}$ of inoculum was added to each tube containing $1 \mathrm{~mL}$ of antimicrobial agent in the dilution range prepared from a stock solution of L-cys $800 \mathrm{mM}$. The inoculated macrodilution tubes were incubated for $24 \mathrm{~h}$ at $37^{\circ} \mathrm{C}$ to assess antibacterial activity. Control tubes of the medium, broth and broth with L-cys, were also incubated.

\subsection{Textile functionalization process}

An oxidation reaction mediated by 2,2,6,6tetramethylpiperidine-1-oxyl radical (TEMPO) (Sigma Aldrich) was performed, in order to break the hydrogen bonds between hydroxyl groups present on the surface of the microfibrils, which allows the connection of new molecules, in this case L-cys, to cotton fibres by the oxidation of C6 hydroxyl groups into carboxylate ones (Gomes et al., 2010; Fukuzumi et al., 2010).

A solution of $0.0125 \%(w / v)$ of TEMPO; $0.1250 \%(w / v)$ of sodium bromide $(\mathrm{NaBr})$ (Sigma Aldrich) and $3.2 \%(\mathrm{v} / \mathrm{v})$ of sodium hydrochloride ( $\mathrm{NaOCl}$ ) (Panreac) was prepared. Then, $2 \mathrm{~g}$ of fabric samples were submersed in $50 \mathrm{~mL}$ of the previous solution and stirred for $60 \mathrm{~min}$. Afterwards, the $\mathrm{pH}$ of the solution was adjusted to 7 and the samples were washed in deionized water.

For the functionalization solution, several L-cys solutions with different concentrations were prepared, $0.6 \mathrm{mg} / \mathrm{mL}$ ( $3 \%$ over the weight of the fibre (owf)), $1 \mathrm{mg} / \mathrm{mL}$ (5\% owf) and $1.4 \mathrm{mg} / \mathrm{mL}(7 \%$ owf) in distilled water according to a liquor ratio of 1:50 ( $1 \mathrm{~g}$ of fabric into $50 \mathrm{~mL}$ of bioactive solution). The samples were incubated in a Mathis dyeing machine, during $60 \mathrm{~min}$ at $80^{\circ} \mathrm{C}$ and stirred at $15 \mathrm{rpm}$. Afterwards, all samples were washed in a $1 \mathrm{~g} / \mathrm{L}$ of a solution of AATCC 1993 Standard Reference Detergent WOB, in four washing cycles performed at $40^{\circ} \mathrm{C}$ during $60 \mathrm{~min}$, a method adapted from the international standard EN ISO 105-C06:2010, followed by drying at $37^{\circ} \mathrm{C}$ for approximately $4 \mathrm{~h}$. The soaping procedure was performed over the samples treated with L-cys prior to the assessment of antibacterial activity in order to give evidence of a durable functionalization effect.

In these conditions, the binding mechanism through which Lcys is linkage to the cotton (cellulosic fibres) is due to the bond established between the amino group of L-cys and the carboxylic group of 6 created by the TEMPO activation, is elucidated in Fig. 1.

\subsection{Assessment of sulfhydryl groups on cotton samples}

\subsubsection{Ellman's reagent test (quantitative determination of free thiol groups)}

The 5,5'-dithio-bis-(2-nitrobenzoic acid), also known as DTNB, or Ellman's reagent (Sigma Aldrich) is very useful to determine and quantify the free -SH groups from L-cys according with the mechanism of Fig. 1. Briefly, the colorimetric method was performed by measuring the absorbance of the functionalization/bioactive 

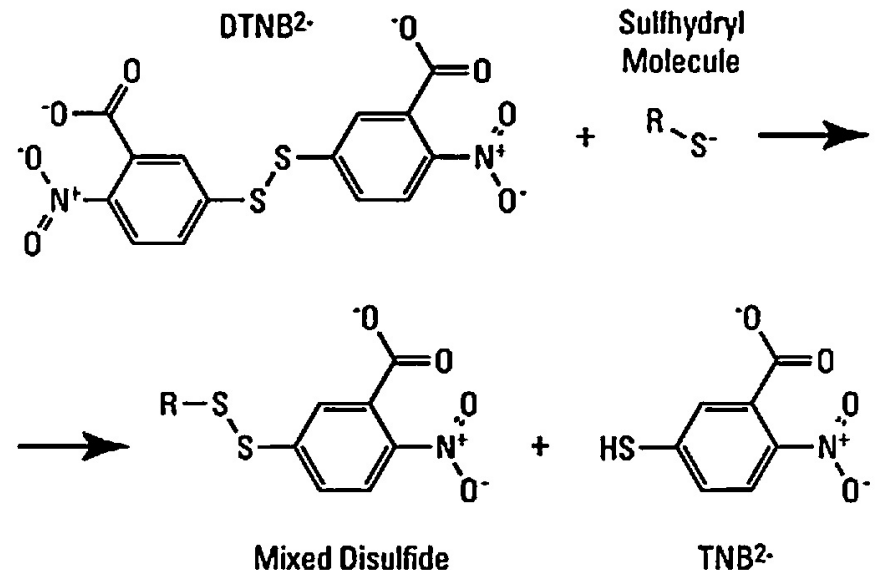

Fig. 1. DTNB mechanism for the detection of sulfhydryl groups of L-cysteine (source: (Protocol Ellman's Reagent, 2011. Thermo Fisher Scientific Inc.-Pierce Biotechnology, USA).

solution at $412 \mathrm{~nm}$ before and after incubation/functionalization, and by estimating the amount of sulfhydryl groups by means of calibration curve (of known concentrations of L-cys). The analysis of free thiol groups was carried out in the solution of the incubation bath, before and after the functionalization process, by measuring the absorbance of the solutions. An exhaustion rate of L-cys was estimated through the difference of the amount before and after functionalization as described in the literature (Protocol Ellman's Reagent, 2011. Thermo Fisher Scientific Inc.-Pierce Biotechnology, USA). Typically, small aliquots were collected from the various incubation baths, and $250 \mu \mathrm{L}$ of these solutions were placed in test tubes containing $2.50 \mathrm{~mL}$ of sodium phosphate and $50.0 \mu \mathrm{L}$ of Ellman's reagent. The tubes were stirred and left to incubate at room temperature for a period of $15 \mathrm{~min}$. Afterwards, the absorbance at $412 \mathrm{~nm}$ was measured for each solution.

\subsubsection{EDX analysis (energy-dispersive $X$-ray spectroscopy)}

The elemental analysis by energy dispersive X-ray spectroscopy (EDX) was conducted to confirm the presence of sulphur (S), and therefore L-cys, in the functionalized samples. This test was performed on cotton samples functionalized with 3,5 and 7\% owf of L-cys and samples without any treatment (control). The detector used is Rontec and is coupled to scanning electronic microscope of Hitachi (model S-2700).

\subsection{Fourier transform infrared spectroscopy (FT-IR) analysis}

The structural changes of the different samples was analysed by FT-IR to give information about the effective linkage of L-cys onto cotton fibres. In addition, in order to eliminate the possible contribution to antibacterial effect of probable physically attached L-cys onto cotton fibres, an additional assay was performed by applying the functionalization processes using $5 \%$ owf of L-cys, as described above, over non pre activated cotton with TEMPO. Moreover, with this assay it was possible to validate the mechanism proposed and established in Fig. 2.

Briefly, non-activated and non-functionalized cotton samples (cotton), cotton activated with TEMPO (cotton activated), functionalized cotton without pre-activation with TEMPO (cotton-L-cys without activation) and activated and functionalized cotton samples (cotton-L-cys with activation) were tested. Measurements were done with Thermo-Nicolet is10 FTIR spectrophotometer. Samples were scanned 64 times each, with a spatial frequency resolution of $4 \mathrm{~cm}^{-1}$.

\subsection{Assessment of the antibacterial activity of the textile samples}

Antibacterial activity of L-cys was tested against two bacterial strains-a Gram positive strain S. aureus (ATCC 6538) and a Gram negative strain K. pneumoniae (ATCC 4352), according to the Japanese Industrial Standard JIS L Standard 1902:2002 (testing for antibacterial activity and efficacy on textile products).

Bacterial inoculums were prepared from an overnight liquid culture in Nutrient Broth (Fluka Analytical) and incubated at $110 \mathrm{rpm}, 37^{\circ} \mathrm{C}$ for $24 \mathrm{~h}$. Bacterial concentrations were then adjusted to $1-2 \times 10^{8}$ cells $/ \mathrm{mL}$ (equivalent to 0.5 McFarland) and working standards were prepared to a final concentration of $1 \pm 0.3 \times 10^{5} \mathrm{CFU} / \mathrm{mL}$. The samples were used to measure the number of live bacteria after inoculation $\left(T_{0 \mathrm{~h}}\right)$ and to measure the number of live bacteria after incubation $\left(T_{24 \mathrm{~h}}\right)$. The fabric samples were placed in a $50 \mathrm{~mL}$ Falcon tubes and $200 \mu \mathrm{L}$ previously prepared of the working standards was added. $T_{24 \mathrm{~h}}$ was derived from tubes incubated for $24 \mathrm{~h}$ at $37^{\circ} \mathrm{C}$ while $T_{0 \mathrm{~h}}$ was measured on initial samples. To release the bacterial cells from the textile samples, before and after the $24 \mathrm{~h}$ incubation period, $20 \mathrm{~mL}$ of $0.85 \%$ $\mathrm{NaCl}$ with surfactant Tween 80 (Fluka Analytical) $(0.2 \%(\mathrm{v} / \mathrm{v}))$ was added to the samples in $50 \mathrm{~mL}$ Falcon tubes. The resulting suspensions were diluted in sterile $0.85 \%$ sodium chloride solution ( $1: 10$; $1: 100 ; 1: 1000(\mathrm{v} / \mathrm{v}))$ and plated to determine the viable counts. The plates were incubated at $37^{\circ} \mathrm{C}$ for $24 \mathrm{~h}$, and the number of colonies was determined. This procedure was performed in triplicate. The growth reduction rate of the bacteria was calculated using the equation:

$$
\frac{T_{0 h}-T_{24 h}}{T_{0 h}} \times 100 \%=\text { reduction rate }
$$

where $T_{0 \mathrm{~h}}$ is the $\mathrm{CFU} / \mathrm{mL}$ of bacterial colonies at the initial stage $(0 \mathrm{~h})$ and $T_{24 \mathrm{~h}}$ is the CFU/mL of bacterial colonies after $24 \mathrm{~h}$ incubation.

\subsection{Evaluation of the cytotoxicity of the functionalized textile samples}

This procedure was performed according to E DIN EN ISO 109935. Initially, it was prepared a perspiration extract of the bioactive material functionalized with an L-cys concentration of $5 \%$ owf, which presented total inhibition of bacterial growth. The bioactive material was incubated with a perspiration solution, for $24 \mathrm{~h}$ at $37^{\circ} \mathrm{C}$ under slight shaking. The resulting perspiration extract was set up to a $\mathrm{pH}$ value of 7.3-7.4 with sodium hydroxide (Panreac) and filtrated in sterile conditions. A cell culture of Connective tissue cells L 929 [ATCC No. CCL1, NCTC clone 929 L (DSMZ), was treated for $68-72 \mathrm{~h}$ with the perspiration extract diluted about $33.3-4.4 \%$. To confirm the validity of the test system, controls were carried out along the experiment. Control tests made included: solvent control (phosphate buffered solution diluted in culture medium corresponding to the test material), positive control (5\% DMSO in culture medium) and negative control (culture medium). Concentrations of the tested material in culture medium were $4.4 \%, 6.6 \%, 9.9 \%, 14.8 \%$, $22.2 \%$, and $33.3 \%$, respectively. After the incubation period, the protein content of the test groups cultures (Culture medium: DMEM with $10 \%$ FBS) was assessed by the bicinchoninic acid (BCA) method and compared with the protein content of the controls. From this analysis, the cell growth was determined in the presence of the test material. In the presence of cell-toxic substances there is a modified proliferation and partition rates of the cells (growth inhibition test) (Gouveia et al., 2011). This leads to a reduction in protein content of the cell culture as compared to the control cultures, which was here analyzed using a colorimetric method (BCA test) after 68-72 h incubation time. Briefly, the protein contents were measured colorimetrically using the BCA reagents (Uptima) and the absorption at $540 \mathrm{~nm}$ was measured using a microplate auto reader. The mean 


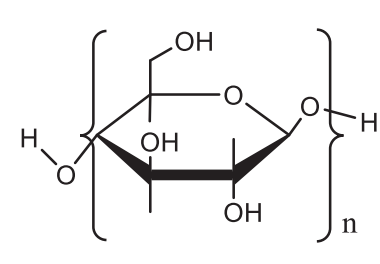

Cellulose

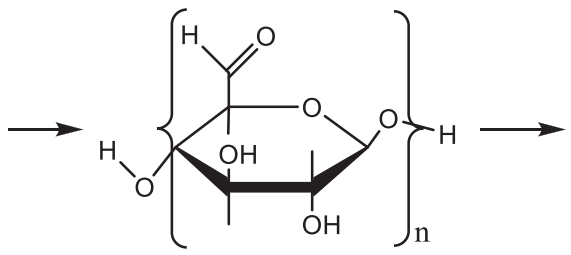

C6-aldehyde

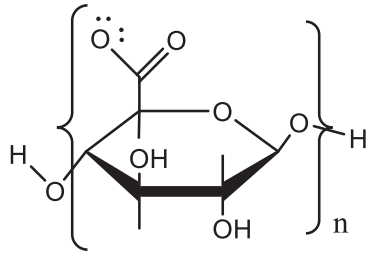

C6-carboxylic acid

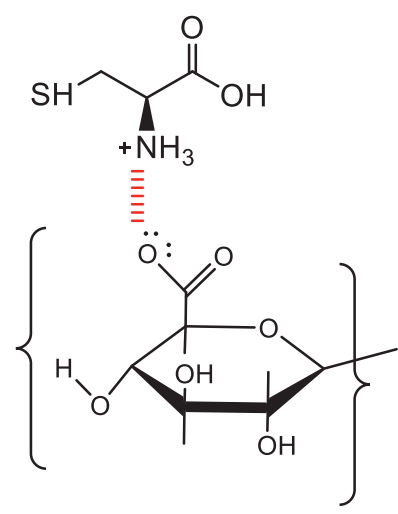

L-cys linked to cellulose

Fig. 2. Proposed mechanism of linkage of L-cysteine onto TEMPO activated cotton samples.

absorption $\left(A_{540 \mathrm{~nm}}\right)$ and standard deviation of three parallel wells (cultures) was calculated and used for assessing the percentage of growth inhibition (G.I.) following the depicted formula:

$\%$ G.I. $=100-100 \times\left(A_{540 \mathrm{~nm}}\right.$ sample $)-\left(A_{540 \mathrm{~nm}}\right.$ blank $)$

$\left(A_{540 \mathrm{~nm}}\right.$ control $)-\left(A_{540 \mathrm{~nm}}\right.$ blank $)$

where $A_{540 \mathrm{~nm}}$ sample is the absorption value of the test extract; $A_{540 \mathrm{~nm}}$ blank is the absorption value of blank cultures (without cells); $A_{540 \mathrm{~nm}}$ control is the absorption value of the solvent control.

\subsection{Study of mechanism of action of L-cys to understand its antibacterial behaviour}

\subsubsection{Exposure of bacteria to L-cys}

In order to establish the best time of contact of the microorganisms with the antimicrobial agent L-cys for further investigation of the mechanism of action through flow cytometry, the death curves for each microorganism were assessed. Suspensions of the microorganisms were obtained from an exponentially growing culture of each bacterial strain in Mueller-Hinton Broth (MHB) (LiofilChem) at $37^{\circ} \mathrm{C}$ and $150 \mathrm{rpm}$. This cell suspension was used to inoculate tubes containing the controls (MHB) and L-cys samples $(1 \times \mathrm{MIC})$, to study the potential bacteriostatic or bactericidal effect of $\mathrm{L}$-cys. The inoculated $50 \mathrm{~mL}$ flasks containing $2.5 \mathrm{~mL}$ of MHB with the appropriate controls and sample concentration were inoculated with $2.5 \mathrm{~mL}$ of the exponentially-grown bacterial cells to yield a final concentration of approximately $1 \times 10^{6} \mathrm{CFU} / \mathrm{mL}$. Flasks were incubated at $37^{\circ} \mathrm{C}$ and aliquots were removed at $0,2,4,6,8$ and $24 \mathrm{~h}$ for the determination of viable counts. Serial dilutions were prepared in sterile $0.85 \%$ sodium chloride solution and plated. The plates were incubated at $37^{\circ} \mathrm{C}$ for 18 to $24 \mathrm{~h}$, and the number of colonies was determined by cell counting (performed in triplicate), and means were calculated (Bouhdid et al., 2010).

\subsubsection{Cytometry analysis}

The contact time established for flow cytometric experiments and plate counting was $2 \mathrm{~h}$, for both strains, evaluated form the death curves above described. After $2 \mathrm{~h}$, samples were centrifuged at $10,000 \mathrm{~g}$ for $10 \mathrm{~min}$, washed and resuspended in $1 \mathrm{~mL}$ sterile PBS. In all cases, control experiments were carried out in parallel wells; cells were incubated in buffered peptone water and treated under the same conditions.

The bacterial concentration for this test was $1 \times 10^{6} \mathrm{CFU} / \mathrm{mL}$ and the staining protocols for the flow cytometry experiments were the following:

(a) For membrane permeability assessment, suspensions of Gram positive and Gram negative cells were incubated with $10 \mu \mathrm{g} / \mathrm{mL}$ of propidium iodide (PI) (Sigma-Aldrich) in PBS buffer (pH 7.4). Gram positive bacterial suspensions were incubated for $5 \mathrm{~min}$ at room temperature and Gram negative bacteria were incubated for $15 \mathrm{~min}$ at $37^{\circ} \mathrm{C}$.

(b) In order to assess metabolic activity, suspensions of Gram positive and Gram negative cells were incubated with $50 \mu \mathrm{M}$ cyanoditolyl tetrazolium chloride (CTC) (Polysciences) in Nutrient Broth and incubated for $60 \mathrm{~min}$ at $37^{\circ} \mathrm{C}$ and $150 \mathrm{rpm}$.

(c) For the evaluation of membrane potential, suspensions of Gram negative cells were incubated with $2.5 \mu \mathrm{g} / \mathrm{mL}$ bis-(1,3-dibutylbarbituric acid) trimethine oxonol (BOX) (Molecular Probes, Invitrogen) in PBS buffer with 4 mM EDTA ( $\mathrm{pH} 7.4$ ). Gram negative bacteria were incubated for $15 \mathrm{~min}$ at $37^{\circ} \mathrm{C}$.

(d) For the staining of Gram positive bacteria, in order to differentiate bacterial cells from noise due to the reduced size of $S$. aureus cells, after labelling with PI and CTC, $10 \mu \mathrm{g} / \mathrm{mL}$ of fluorescein isothiocyanate (FITC) (Sigma-Aldrich) was added to each cell 
Table 1

Determination of the minimal inhibitory concentration of L-cys for the studied strains (run in triplicate).

\begin{tabular}{|c|c|c|c|c|c|c|c|c|c|}
\hline & & & & & L-Cys soluti & & & & \\
\hline Concentration $(\mathrm{g} / \mathrm{mL})$ & $9.7 \times 10^{-2}$ & $4.8 \times 10^{-2}$ & $2.4 \times 10^{-2}$ & $1.2 \times 10^{-2}$ & $6.0 \times 10^{-3}$ & $3.0 \times 10^{-3}$ & $1.5 \times 10^{-3}$ & $1.9 \times 10^{-4}$ & $2.3 \times 10^{-5}$ \\
\hline S. aureus & - & - & + & + & + & + & + & + & + \\
\hline K. pneumoniae & - & - & - & - & - & + & + & + & + \\
\hline
\end{tabular}

- Without turbidity (microorganism growth inhibition).

+ With turbidity.

Table 2

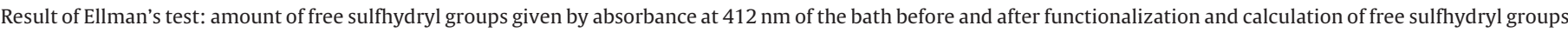
concentration on cotton samples (run in triplicate).

L-cys (owf) (amount used in function of cotton samples) $(\%)$
L-cys Concentration used for functionalization $(\mathrm{mg} / \mathrm{mL})$
L-cys Concentration in bath after functionalization $(\mathrm{mg} / \mathrm{mL})$
L-cys Concentration impregnated in cotton samples $(\mathrm{mg} / \mathrm{mL})$

suspension and incubated for $15 \mathrm{~min}$ at room temperature. The cell suspensions were washed twice in PBS buffer (pH 7.4) and resuspended in the same buffer.

(e) Exponentially-grown cells $\left(18 \mathrm{~h}\right.$ at $\left.37^{\circ} \mathrm{C}, 150 \mathrm{rpm}\right)$ were used as negative control for PI and BOX staining protocols and as positive control for CTC staining.

(f) Prior to the flow cytometric analysis, all cell suspensions were washed once in PBS buffer ( $\mathrm{pH}$ 7.4) and resuspended in the same buffer. Experiments were performed in duplicate.

Bacterial samples were analysed on a CyAn ADP (Beckman Coulter Inc.). Acquisition was performed with Summit Software (Beckman Coulter Inc.). The acquisition was based on light scatter and fluorescence signals resulting from $15 \mathrm{~mW}$ laser illumination at $488 \mathrm{~nm}$. Light scatter measurements and fluorescence measurements were acquired logarithmically. Signals corresponding to forward and side scatter (FS and SS) and fluorescence were accumulated. Threshold levels were set on SS to eliminate noise or particles much smaller than intact cells with the exception of FITC-labelled S. aureus cells where the threshold level was set on FITC. Bacterial cells were gated according to FS/SS parameters. Sample acquisition was performed at low flow rate setting, a total of 10,000 events were acquired for each sample. Data analysis was performed using FCS Express version 4 Plus Research Edition (De Novo Software).

\section{Results}

\subsection{Determination of the minimal inhibitory concentration}

The results presented in Table 1 show the MIC values for $K$. pneumoniae of $48 \mathrm{mg} / \mathrm{mL}$, and for $S$. aureus of $6 \mathrm{mg} / \mathrm{mL}$. Turbidity was observed when lower concentrations were used revealing no inhibitory effect of the growth of the microorganisms, in these cases.

\subsection{Assessment of sulfhydryl groups on cotton samples}

\subsubsection{Ellman's reagent test (quantitative determination of free thiol groups)}

From the calibration curve obtained in the Ellman's test, the amount of free -SH groups present in each functionalization bath before and after functionalization was calculated. The amount of free sulfhydryl groups present in the cotton samples after functionalization was calculated subtracting the concentration value before and after the functionalization process, using the equation of the calibration curve to calculate the amount of free $\mathrm{SH}$ groups present in the cotton samples (Table 2).

\subsubsection{FT-IR Analysis}

The IR spectra clearly suggest the presence and fixation of Lcys onto cotton fibres via the pre-activation with TEMPO according with the mechanism shown in Fig. 2. In contrast in the case of the sample $C$ (non-activated cotton functionalized with L-cys) the spectra clearly shows that no cysteine was linked onto cotton fibres since it was not possible to detect the S-H stretching at $2599 \mathrm{~cm}^{-1}$.

Structural changes on cotton before and after activation and/or functionalization were also assessed by FT-IR. Fig. 3 shows the four spectra for the different samples prepared as follows: Sample A correspond to unmodified cotton, sample B to cotton activated with TEMPO, sample $C$ to non-activated cotton functionalized with L-cys and sample $\mathrm{D}$ to activated and functionalized cotton.

The IR spectrum of unmodified cotton (sample A) presented the expected characteristic peak at $3330 \mathrm{~cm}^{-1}$ due to $\mathrm{O}-\mathrm{H}$ stretching vibrations, and at $1335 \mathrm{~cm}^{-1}$ assigned to $\mathrm{O}-\mathrm{H}$ deformation vibrations. The specific bands of the $\beta(1-4)$ glycoside bridge at 1159 and $897 \mathrm{~cm}^{-1}$, as well as $\mathrm{C}-\mathrm{O}-\mathrm{C}$ stretching vibration in the pyranose ring at $1030 \mathrm{~cm}^{-1}$ (Socrates, 2004) are also clearly present in the spectra.

In the case of sample $B$, the spectrum shows a peak around $1600 \mathrm{~cm}^{-1}$ correspondent to the ionic form of the carboxylate that was bounded to cotton fibres through the activation with TEMPO (Gomes et al., 2012). This IR spectrum definitely confirms the effect of the activation process to further linkage of L-cys via the exhaustion method that was developed with success.

The presence of L-cys was confirmed in sample D (activated and functionalized cotton) with observed peak of amides, namely vibrations of amide $I$ band due to $C=O$ stretching at $1650 \mathrm{~cm}^{-1}$, $\mathrm{N}-\mathrm{H}$ bending of primary amines at $1587 \mathrm{~cm}^{-1}$, and the amide III at $1340 \mathrm{~cm}^{-1}$ (C-N stretching vibrations); Also $-\mathrm{CH}_{3}$ symmetrical angular deformation is showed at $1382 \mathrm{~cm}^{-1}$. More importantly, the spectrum of cotton-L-cys also presented the S-H stretching of $-\mathrm{CH}_{2} \mathrm{SH}$ functional groups at $2599 \mathrm{~cm}^{-1}$ (Socrates, 2004). In contrary, sample C (with L-cys and without TEMPO) does not show evidence of the presence of L-cys according to the absence of the above described characteristic peaks.

Table 3

Results of the effect of L-cys on bacterial reduction (\%) of cotton samples, analysis of cys soaked textiles.

\begin{tabular}{lllccc}
\hline S. aureus & \% L-cys (owf) & $0 \%$ & $3 \%$ & $5 \%$ & $7 \%$ \\
& \% Reduction rate & 0 & 59.09 & 85.33 & 52.12 \\
K. pneumonia & \% L-cys (owf) & $0 \%$ & $3 \%$ & $5 \%$ & $7 \%$ \\
& \% Reduction rate & 0 & 44.00 & 83.33 & 10.26 \\
\hline
\end{tabular}



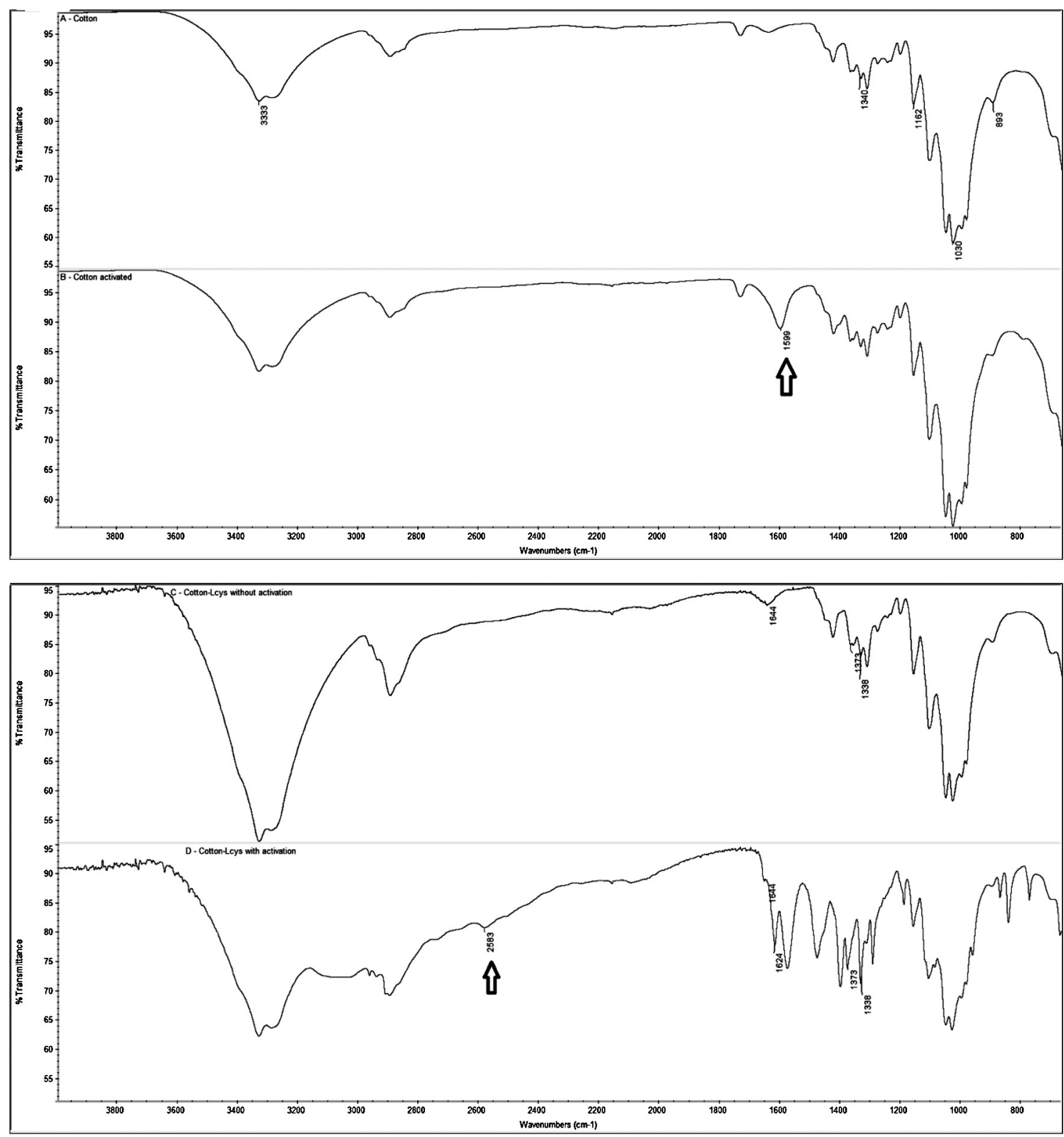

Fig. 3. FT-IR analysis of the different stages of the non-activated and activated cotton further functionalized with L-cys (run in three parallel samples).

\subsubsection{EDX analysis (energy-dispersive X-ray spectroscopy)}

An analysis by EDX (energy-dispersive X-ray spectroscopy) was performed on functionalized cotton samples to check for the presence of sulphur in the samples.

As it can be seen in Fig. 4, there are $S$ groups in the functionalized samples, showing L-cys presence in those samples.

\subsection{Assessment of the anti-bacterial activity of the textile samples}

Antimicrobial tests showed a decrease in bacterial growth for the two strains at different concentrations (Table 3), as desirable.

For S. aureus, it can be seen in Table 3 a growth reduction of $59.09 \%, 85.33 \%$ and $52.12 \%$ for samples containing $3 \%, 5 \%$ and $7 \%$ (w/w) of L-cys, respectively. For the K. pneumoniae strain, a growth reduction of $44.00 \%, 83.33 \%$ was visible for samples containing $3 \%$ and $5 \%(\mathrm{w} / \mathrm{w})$ of L-cys, respectively; whereas only $10.26 \%$ growth reduction was obtained for the samples with the highest L-cys concentration, $7 \%(\mathrm{w} / \mathrm{w})$.

\subsection{Exposure of bacteria to L-cys: Evaluation of the time of death}

The contact of L-cys with the strains in study allowed to monitor the effect of L-cys on the bacteria. This assay was performed for a period of $24 \mathrm{~h}$, allowing the construction of death curves (Fig. 5).

In the graphs of the death curves (Fig. $5 \mathrm{~A}$ and $\mathrm{B}$ ) it can be seen that there is not a complete inhibition of bacterial growth (death). It can only be seen a decrease of growth and at $24 \mathrm{~h}$ it can be verified that for both strains there are a more pronounced reduction of growth at $2 \mathrm{~h}$ of incubation. This behaviour is normal when bacteriostatic effect is present since culture medium can still feed the remaining survivors and the $24 \mathrm{~h}$ was not enough to run out of nutrients as reported and found elsewhere (Klancnik et al., 2010). Likewise, the effect of L-cys over both strains should be a bacteriostatic, since there is maintenance of the number of $\mathrm{CFU} / \mathrm{mL}$ of the initial inoculum $\left(1 \times 10^{6} \mathrm{CFU} / \mathrm{mL}\right)$. In order to consider the activity of L-cys as a bactericide over those bacteria it would be necessary that the reduction was $99.9 \%$ of total $\mathrm{CFU} / \mathrm{mL}$ of initial inoculum. 


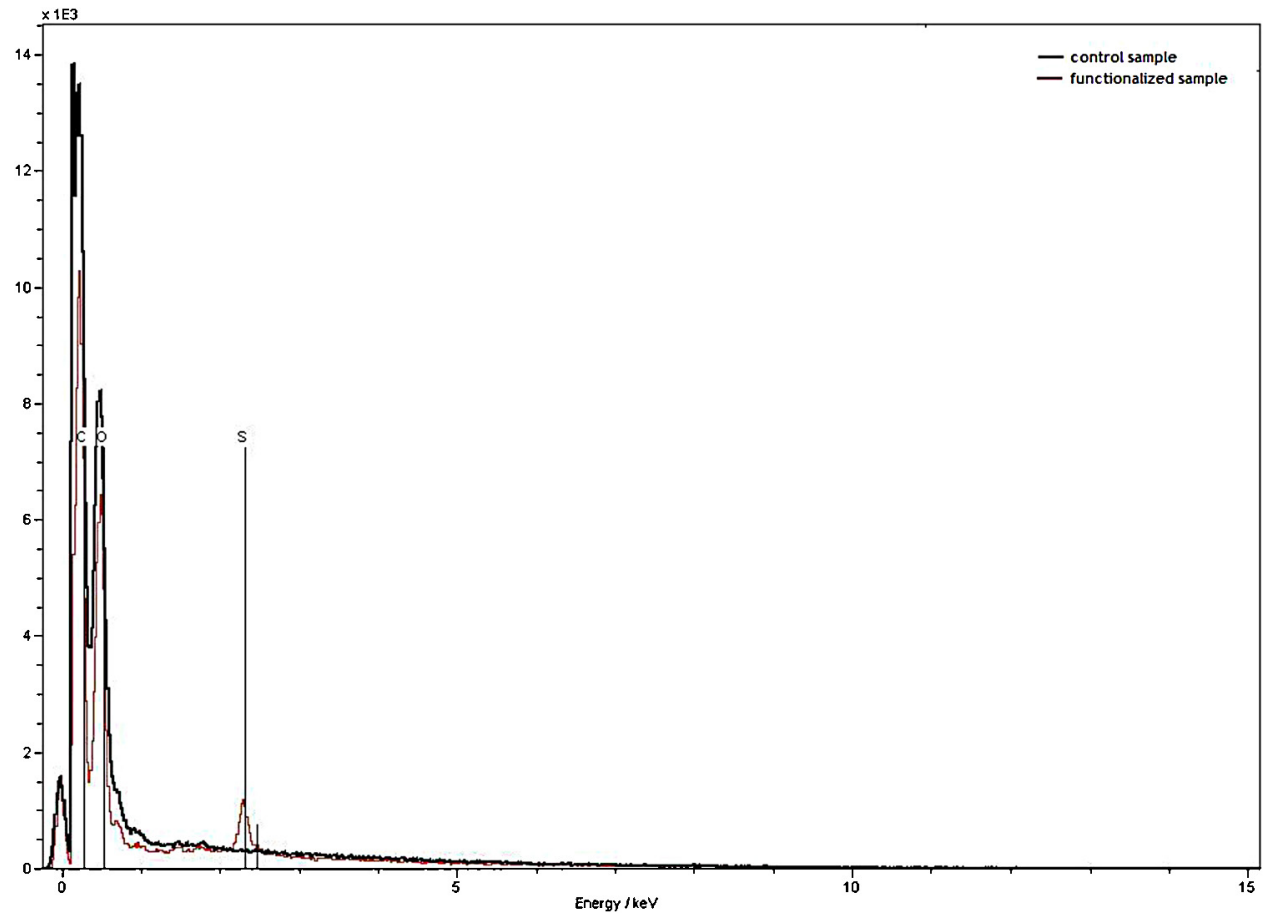

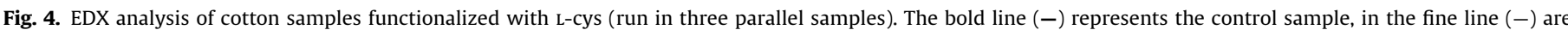
represented the functionalized samples, analysis of cys soaked textiles.
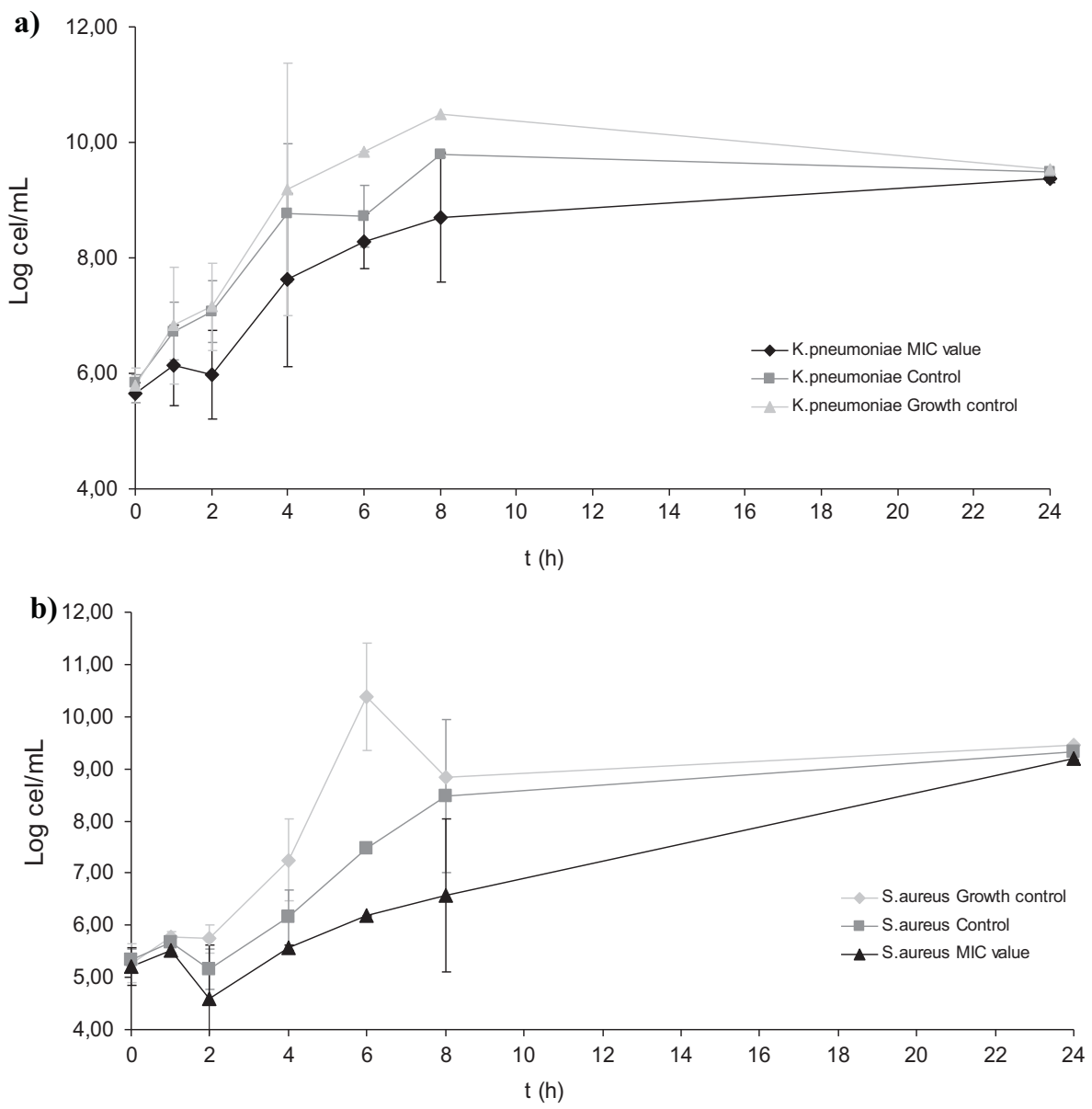

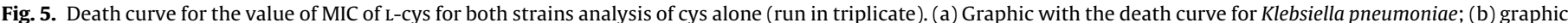

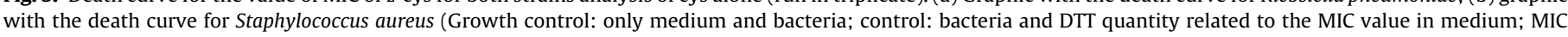
value: bacteria and L-cys in concentration corresponding with the MIC value). 
Table 4

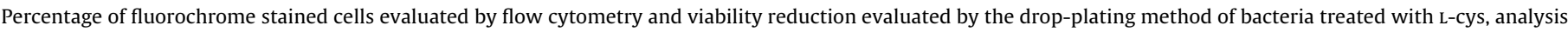
of cys alone (run in triplicate).

\begin{tabular}{|c|c|c|c|c|c|}
\hline & Sample & Viability reduction (\%) & PI positive cells (\%) & CTC positive cells (\%) & BOX positive cells (\%) \\
\hline Klebsiella & Positive control & & 0.38 & 97.39 & 13.74 \\
\hline \multirow[t]{3}{*}{ pneumoniae } & Negative control & 100 & 65.56 & 11.54 & 75.75 \\
\hline & Control & & $0.65 \pm 0.19$ & $94.26 \pm 1.77$ & $4.20 \pm 0.12$ \\
\hline & MIC & $79.88 \pm 1.94$ & $0.90 \pm 0.55$ & $2.62 \pm 0.37$ & $8.05 \pm 0.60$ \\
\hline Staphylococcus & Positive control & & 1.89 & 67.13 & \\
\hline \multirow{3}{*}{ aureus } & Negative control & 100 & 67.28 & 18.75 & \\
\hline & Control & & $2.73 \pm 1.15$ & $91.08 \pm 6.84$ & \\
\hline & MIC & $98.9 \pm 1.13$ & $1.88 \pm 0.09$ & $1.46 \pm 0.53$ & \\
\hline
\end{tabular}

\subsection{Study of mechanism of action}

Flow cytometry tests for bacteria in suspension were conducted using the MIC value obtained for each bacteria. The results obtained for K. pneumoniae (Table 4) showed that there is a small membrane depolarization (BOX positive cells) in cells incubated with L-cys; however, the primary mechanism of action of L-cys on Gram negative bacteria seems to be related with metabolic activity alterations, since there is a streak decrease in the number of metabolic active cells (CTC positive cells) after incubation with L-cys with a reduction of about $94.26 \%$ to $2.62 \%$ CTC positive cells. The changes on PI labelling showed no significant alterations to consider any effect on bacterial membrane permeabilization (PI positive cells) which could result in bacterial cell death.

\subsection{Cytotoxicity analysis}

Results for the cytotoxicity analysis are shown in Table 5. The perspiration extract of the test material (functionalized with $5 \%$ (w/w) of L-cys) showed a growth inhibition (G.I.) of $25 \%$ in the cytotoxicity test under the mentioned conditions.

\section{Discussion}

\subsection{Determination of the minimal inhibitory concentration}

The different MIC for the two strains ( $S$. aureus and K. pneumoniae) can be due to the fact that most antimicrobials act easier against Gram positive ( $S$. aureus) than against Gram negative (K. pneumoniae) because of the composition of Gram negative outer membrane. Typically, the cellular wall of gram-negative bacteria is more complex than that of the gram-positive bacteria

\section{Table 5}

Cytotoxicity results for the tested item (functionalized cotton with 5\% L-cys) (run in triplicate).

\begin{tabular}{llll}
\hline $\begin{array}{l}\text { Rel. protein content }\left(\mathrm{A}_{540}\right. \\
\text { three parallel cultures) }\end{array}$ & Mean & Standard deviation & G.I.\% \\
\hline Blank & 0.221 & \pm 0.003 & \\
Positive control & 0.185 & \pm 0.070 & 100 \\
Negative control & 1.180 & \pm 0.020 & 0 \\
Solvent control (\%) & & & \\
33.30 & 1.118 & \pm 0.021 & 0 \\
22.20 & 1.127 & \pm 0.065 & 0 \\
14.80 & 1.164 & \pm 0.074 & 0 \\
9.90 & 1.118 & \pm 0.031 & 0 \\
6.60 & 1.179 & \pm 0.025 & 0 \\
4.40 & 1.180 & \pm 0.073 & 0 \\
Test extract $(\%)$ & & & 25 \\
33.30 & 0.890 & \pm 0.059 & 6 \\
22.20 & 1.074 & \pm 0.031 & 0 \\
14.80 & 1.160 & \pm 0.033 & 0 \\
9.90 & 1.123 & \pm 0.090 & 0 \\
6.60 & 1.189 & \pm 0.070 & 0 \\
4.40 & 1.206 & \pm 0.071 & \\
\hline
\end{tabular}

presenting itself as a layered structure. Whereas the innermost layer is more rigid and essentially constituted by peptidoglycan, an outer layer, absent in the wall of Gram positive bacteria, known as outer membrane is composed of lipopolysaccharides, phospholipids, and proteins. The outer membrane is responsible for the inherently resistance of these organisms to antimicrobial compounds (Maillard, 2002; Prescott et al., 2008), being in accordance with the results for the $K$. pneumoniae presented in our study.

These MIC values are significantly higher in comparison with others obtained for example for chitosan $(0.05 \%(\mathrm{w} / \mathrm{v})$ against $S$. aureus and K. pneumonia) and for triclosan (6\% (w/v) against $S$. aureus) (Kim et al., 2003; Cottell et al., 2009). However, the ability of L-cys for crosslinking once in an aqueous solution might have an important role in these results since a higher concentration should be required to obtain the sulfydryl groups in a free form with the consequent antibacterial activity. Though, once in the cotton fibres, the immobilization requires less concentrated L-cys solutions to give cotton fibres the desired antibacterial activity, as discussed below.

\subsection{Assessment of sulfhydryl groups on cotton samples}

\subsubsection{Ellman's reagent test (quantitative determination of free thiol groups)}

As can be seen by the values obtained (Table 2), the samples with a higher concentration of free - $\mathrm{SH}$ groups are the ones that were functionalized with $1.0 \mathrm{mg} / \mathrm{mL}$ ( $5 \%$ owf), since the number of $-\mathrm{SH}$ groups decreased in the samples functionalized with $1.4 \mathrm{mg} / \mathrm{mL}$. This result was due to the fact that L-cys molecules react with each other in solution or precipitate, being less available to bind to cotton as discussed elsewhere (Gouveia et al., 2011).

\subsubsection{FT-IR analysis}

Structural changes on cotton before and after activation and/or functionalization were also assessed by FT-IR analysis in order to give evidence of effective linkage and durable presence of L-cys after activation and functionalization. The correspondent IR spectrum (sample D) definitely confirms the effective linkage of L-cys via the exhaustion method that was performed with success over pre-activated cotton samples with TEMPO. Results clearly put in evidence that pre-activation of cotton with TEMPO is imperative for an effective and durable linkage of L-cys onto cotton fibres, as expected.

\subsection{Study of anti-bacterial activity in textile samples}

As it can be seen in Table 3, the phenomenon of the growth reduction can also be observed by the results of Ellman's test, where there is a higher presence of free $\mathrm{SH}$ groups on the samples with 3 and 5\% owf of L-cys than in the samples functionalized with 7\% $(\mathrm{w} / \mathrm{w})$. The fact that the reduction in growth for the samples with $7 \%(w / w)$ L-cys was less than the samples with $5 \%(w / w)$, could be related with the possible precipitation of L-cys. The amino acid in 
solution can form aggregates by forming disulfide bonds between molecules of L-cys, decreasing the availability of these groups to interact with bacteria, such as demonstrated in the present and previous work (Gouveia et al., 2011).

In addition, these growth reduction values demonstrated that L-cys has the ability to cause a marked decrease in bacterial growth (85.33\% for S. aureus and $83.33 \%$ K. pneumoniae) with L-cys concentrations in cotton samples of approximately $\left(0.27 \times 10^{-3} \mathrm{~g} / \mathrm{mL}\right.$ amount of L-cys on cotton fibres after the functionalization process with $5 \%$ owf of L-cys) which is a much lower value than the MIC obtained for each strain (K. pneumoniae and S. aureus $48 \times 10^{-3}$ and $6.0 \times 10^{-3} \mathrm{~g} / \mathrm{mL}$, respectively). The possible reason for the lower $\mathrm{L}^{-}$ cys concentrations required to promote bacterial inhibition when the amino acid is bounded to the fibres can be related with the fact that the free thiol groups are more available in their free form, giving a higher antibacterial effect.

The different inhibitory behaviour of textiles against the two bacteria strains could be related to the effect of L-cys in different bacterial strains, due to the membrane composition as has been discussed above in the discussion of the obtained MIC values (Section 3).

Therefore, the functionalization of cotton fibres with L-cys was obtained with success showing the ability of giving antimicrobial properties to cotton materials.

\subsection{Study of mechanism of action of L-cys to understand its antibacterial behaviour}

Despite the fact that the death curves evaluated in order to check for the best time of contact of L-cys with the studied microorganisms prior to further evaluation on flow cytometry, presented some high deviations in some points (hours), the results from flow cytometry showed that the $2 \mathrm{~h}$ contact selected to perform the assays was very accurate, since it was enough to establish the mechanism of action of L-cys onto bacteria. Regarding the results obtained for FITC-labelled S. aureus (Table 4), similarly to K. pneumoniae, there is a marked reduction in metabolic activity from approximately $91.08 \%$ metabolic active cells to about $1.46 \%$, which is lower than the reduction observed for $K$. pneumoniae. Also for S. aureus, the percentage of PI positive cells is low and therefore, no cell death effect can be considered. With those results it can be stated that the main mechanism of action of L-cys on both bacteria, K. pneumoniae and $S$. aureus, is the reduction in metabolic activity, consistent with the results that confirm the effect of L-cys on both these bacteria is bacteriostatic, as could be seen by the death curves. In fact, changes in metabolic activity can cause permanent damage to prevent bacterial growth and these results are the first to our knowledge that clearly shows the mechanism of action of the antibacterial effect of $\mathrm{L}$-cys on Gram positive and Gram negative bacteria, in particular $K$. pneumoniae and S. aureus.

\subsection{Cytotoxicity analysis}

Changes of cell proliferation due to the presence of cytotoxic substances were analyzed in a cell growth inhibition test by comparing the protein content of the cell cultures treated with an extract of the test items with that of the untreated controls. In the present study growth analysis of cell cultures with test extracts showed no relevant growth inhibition of L929 cells. In addition, the controls confirmed the validity of the study. Cell growth of the positive cultures was inhibited by $100 \%$ and the extract of the negative control did not show any inhibition of cell growth (0\%) (Table 5 ).

A growth inhibition of more than $30 \%$ in comparison with the solvent control is assessed as a clear cell-toxic effect. Therefore, the cytotoxicity test for $5 \%$ over weight of the fabric of L-cys revealed less than $30 \%$ of cellular viability reduction (G.I. $=25 \%$ ), making L-cys a safe antimicrobial agent (Borenfreund and Borrero, 1984).

In accordance with this, it can be concluded that no leachable substances are released from the functionalized cotton materials in cytotoxic concentrations.

\section{Conclusions}

With this study it was possible to develop a new antimicrobial finishing process for cotton or other cellulosic fibres or materials, being non-toxic and environment friendly, unlike most commonly used processes for this purpose.

The new process using L-cys was developed with success to give cotton antimicrobial properties in which L-cys has an antibacterial effect against the two strains under investigation. In addition, immobilization of L-cys onto textiles showed an enhancement in the antibacterial activity by maintaining the free bioactive groups that interact with the microorganism membranes.

Flow cytometry allowed to establish the mechanism by which the groups SH induce growth inhibition. The main mechanism of $\mathrm{L}$ cys against $K$. pneumoniae and $S$. aureus is the occurrence of changes in metabolic activity of these bacteria, which is of great scientific importance because it contributes to the innovative and clear knowledge about the L-cys mechanism of action against Gram positive and Gram negative bacteria. To authors knowledge this is the first report that clearly establishes the mechanism of action of L-cys against Gram-negative and Gram-positive bacteria and the benefits of using L-cys as an antimicrobial agent for cotton fibres being eco-friendly and non-toxic.

\section{Acknowledgements}

The authors would like to thank Fundação para a Ciência e Tecnologia (FCT) for the funding granted concerning the project-PTDC/EBB-BIO/113671/2009 (FCOMP-01-0124-FEDER014752) Skin2Tex. We also would like to thank Fundo Europeu de Desenvolvimento Regional (FEDER) through COMPETE-Programa Operacional Factores de Competitividade (POFC) for the cofunding.

We also would like to acknowledge the contribution of Sílvia Nunes, from UBI, for drawing the mechanism presented in Fig. 1.

\section{References}

Ankri, S., Mirelman, D., 1999. Antimicrobial properties of allicin from garlic. Microbes and Infections 1 (2), 125-129.

Borenfreund, E., Borrero, O., 1984. In vitro cytotoxicity assays: potential alternative to the Draize ocular allergy test. Cell Biology and Toxicology 1, 5-65.

Borkow, G., Gabbay, J., 2008. Biocidal textiles can help fight nosocomial infections. Medical Hypotheses 70, 990-994.

Bouhdid, S., Abrini, J., Amensour, M., Zhiri, A., Espuny, M.J., Manresa, A., 2010. Functional and ultra structural changes in Pseudomonas aeruginosa and Staphylococcus aureus cells induced by Cinnamomum verum essential oil. Journal of Applied Microbiology, 1-11.

Cottell, A., Denyer, S.P., Hanlon, G.W., Ochs, D., Maillard, J.-Y., 2009. Journal of Hospital Infections 72,71

Fukuzumi, H., Saito, T., Okita, Y., Isogai, A., 2010. Thermal stabilization of TEMPO oxidized cellulose. Polymer Degradation and Stability 95 (9), 1502-1508.

Gao, Y., Cranston, R., 2008. Recent advances in antimicrobial treatments of textiles. Textile Research Journal 78, 60-72.

Gomes, A.P., Mano, J.F., Queiroz, J.A., Gouveia, I.C., 2010. Assessment of bacteria-textile interactions using scanning electron microscopy: a study on LbL chitosan/alginate coated cotton. Microscopy: Science, Technology, Applications and Education (1), 286-292

Gomes, A.P., Mano, J.F., Queiroz, J.A., Gouveia, I.C., 2012. Layer-by-layer deposition of antibacterial polyelectrolytes on cotton fibres. Journal of Polymers and the Environment 20, 1084-1094

Gouveia, I.C., 2010. Nanobiotechnology: a new strategy to develop non-toxic antimicrobial textiles. Journal of Applied Microbiology, 407-414

Gouveia, I.C., Sá, D., Henriques, M., 2011. Functionalization of wool with L-cysteine: process characterization and assessment of antimicrobial activity and cytotoxicity. Journal of Applied Polymer Science 124 (2), 1352-1358. 
International Standard CLSI M7-A6. 23 (I), 2003. Metodologia dos testes de sensibilidade a agentes antimicrobianos por diluição para bactérias de crescimento aeróbio, 6- a $^{a}$ edição. ISBN 1-56238-486-4.

JISL (Japanese Industrial Standard), 2008. Testing for Antibacterial Activity and Efficacy on Textile Products, 1902-2008.

Joshi, M., Ali, S.W., Purwar, R., 2009. Ecofriendly antimicrobial finishing of textiles using bioactive agents based on natural products. Indian Journal of Fiber Textile Research 34, 295-304.

Kari, C., Nagy, Z., Kovács, P., Hernádi, F., 1971. Mechanism of the growth inhibitory effect of cysteine on Escherichia coli. Journal of General Microbiology 68, 349-356.

Kim, Y.H., Nam, C.W., Choi, J.W., Jang, J.J., 2003. Journal of Applied Polymer Science $88,1567$.

Klancnik, A., Piskernik, S., Jersek, B., Mozina, S.S., 2010. Evaluation of diffusion an dilution methods to determine the antibacterial activity of plant extracts. Journal of Microbiological Methods 81 (2), 121-126.

Lim, S.H., Hudson, S.M., 2003. Review of chitosan and its derivatives as antimicrobial agents and their uses as textile chemicals. Journal of Macromolecular Science-Polymer Reviews 43 (2), 223-269.

Maillard, J.Y., 2002. Bacterial target sites for biocide action. Journal of Applied Microbiology Symposium 92 (Suppl.), 16-27.
Nelson, D.L., Cox, M.M., 2008. Lehninger: Principles of Biochemistry, fifth ed. W.H. Freeman and Company, New York, pp. 860-894 (Chapter 22).

Prescott, Harley, 2008. Klein Microbiology, seventh ed. McGraw-Hill, New York.

Protocol Ellman's Reagent, 2011. Thermo Fisher Scientific Inc.-Pierce Biotechnology, USA.

Ramachandran, T., Rajendrakumar, K., Rajendran, R., 2004. Antimicrobial textiles-an overview. Journal of the Institution of Engineers 84 (2), $42-47$.

Ristić, T., Zemljič, L.F., Novak, M., Kunčič, M.K., Sonjak, S., Cimerman, N.G., Strnad, S., 2011. Antimicrobial efficiency of functionalized cellulose fibers as potential medical textiles. Health Care, 36-51.

Schindler, W.D., Hauser, P.J., 2005. Chemical Finishing of Textiles. The Textile Institute, Woodhead Publishing Ltd, CRC, Boca Raton, FL, pp. 165-174 (Chapter 15).

Socrates, G., 2004. Infrared and Raman Characteristic Group Frequencies: Tables and Charts. John Wiley \& Sons, Ltd, Hoboken, NJ.

Son, Y., Kim, B., Ravikumar, K., Lee, S., 2006. Polymer Imparting durable antimicrobial properties to cotton fabrics using quaternary ammonium salts through 4-aminobenzenesulfonic acid-chloro-triazine adduct. European Polymer Journal 42, 3059-3067. 